\title{
The relation between size and apparent heaviness
}

\author{
DAVID V. CROSS and LAURENCE ROTKIN \\ State University of New York at Stony Brook, Stony Brook, New York 11794
}

\begin{abstract}
A formula for the size-weight illusion was derived from the Stevens and Rubin (1970) finding that heaviness functions form a family of power functions that converge at a common point in the vicinity of the heaviest weight that can be lifted. Magnitude estimations of the apparent heaviness of 42 plastic cylinders varying in size and weight were obtained from 20 subjects, who were allowed to use both hands to lift the weights. It was predicted that this would increase the maximum weight that could be lifted, which would, in turn, enhance the magnitude of the illusion (the dependence of heaviness on size). The results supported this and other predictions of the model concerning the dependence of the illusion on weight as well as volume.
\end{abstract}

Two objects of equal weight may not appear equally heavy if they are of different size, the smaller of the two usually appears to be heavier. This so-called "size-weight illusion" is exemplified by the old catch, "Which is heavier, a pound of lead or a pound of feathers?" Although a pound is a pound, the lead weight invariably feels heavier, as much as three or four times heavier.

The reason the apparent heaviness of an object depends on its perceived size, according to Woodworth (1921), is that one expects larger objects to be heavier and is prepared to exert more effort to lift them; smaller, presumably lighter, objects should require less effort to lift. When the objects are in fact the same weight, the difference in motor preparedness or in one's expectations contributes to different experiences in lifting them. In Woodworth's own words: "What seems to happen ... is a motor adjustment for the apparent weights, as indicated by their visual appearance, with the result that the weight of larger size is lifted more strongly than the weight of smaller size; so that the big one comes up easily and seems light, the little one slowly and seems heavy" (p. 460).

This illusion was given precise quantification over large ranges of weight and volume by J. C. Stevens and Rubin (1970). Using the method of magnitude estimation. they found that apparent heaviness cannot be expressed as a single power function of weight as suggested by S. S. Stevens and Galanter (1957); rather, a family of power functions seems to be needed. For weights of equal volume, heaviness appears to increase as a simple power function of weight. but with changes in volume both the exponent and the proportionality constant of the individual functions changes. The heaviness of an object is a power function of its weight, $W$, with an exponent and constant of proportionality both dependent on the

This research was supported in part by Grant SO-41125 from the National Science Foundation. object's volume, $\mathrm{V}$, as expressed in the following equation:

$$
H(W, V)=f(V) W^{B(V)},
$$

where the size of the exponent, $g(V)$, increases with volume and the constant of proportionality, $f(V)$, decreases as volume increases; the constants are both expressed as different functions of physical volume.

J. C. Stevens and Rubin discovered by extrapolation of the separately fitted functions in this family of curves that they tended to converge at a common point in the vicinity of the heaviest weight that could be lifted under the experimental conditions. This point with coordinates $\left(\mathrm{H}_{\mathrm{c}}, \mathrm{W}_{\mathrm{c}}\right)$ is a common point, or point of concurrence, for all functions in the family. For objects with weight $W_{c}$, all volumes give, apart from random error, the same heaviness $\mathrm{H}_{\mathrm{c}}$, but as $\mathrm{W}$ moves away from this maximum value the rate of change of $\mathrm{H}$ with respect to $\mathrm{W}$ is different for each volume $\mathrm{V}$. If we evaluate Equation 1 at the point of concurrence, we obtain

$$
\mathrm{H}_{c}=\mathrm{f}(\mathrm{V}) \mathrm{W}_{\mathbf{c}}^{\mathrm{g}(\mathrm{V})} \text {. }
$$

A significant consequence of Equation 2 is that the exponent and proportionality constants for the family of curves defined by Equation 1 are simply and mathematically related as follows:

$$
g(V)=b \log f(V)+a
$$

where

$$
b=-\left(\log W_{c}\right)^{-1} \text { and } a=\left(\log H_{c} / \log W_{c}\right) .
$$

From what has been given, it is easy to show that relative heaviness is a simple power function of relative weight as represented by the equation

$$
H(W, V) / H_{c}=\left(W / W_{c}\right)^{g(V)}
$$


which can be rewritten in the equivalent forms

$$
H(W, V) / H_{c}=\left(W / W_{c}\right)^{a-\left[\log f(V) / \log W_{c}\right]}
$$

and

$$
H(W, V) / H_{c}=\left(W / W_{c}\right)^{a}[f(V)]^{\left[1-\left(\log W / \log W_{c}\right)\right]} .
$$

In words, the heaviness of an object relative to the estimated heaviness of the effective maximum weight. $\mathrm{W}_{\mathrm{c}}$, can be expressed as a power product of two terms: one involves its weight relative to $\mathrm{W}_{\mathrm{c}}$, and the other involves an appropriate function of its volume, $f(V)$. Said another way, the equation for heaviness can be factored into two components: one is independent of volume, the other is strictly dependent on volume.

A general equation for the size-weight illusion can be derived from Equation 4 by defining the heaviness ratio of two objects having equal weight, $W$, and volumes $V_{1}$ and $V_{2}$ as follows:

$$
H\left(W, V_{1}\right) / H\left(W, V_{2}\right)=\left(W / W_{c}\right)^{g\left(V_{1}\right)-g\left(V_{2}\right)}
$$

By substituting Equation 3 in Equation 5, we can write this equation in the equivalent forms

$$
\mathrm{H}\left(\mathrm{W}, \mathrm{V}_{1}\right) / \mathrm{H}\left(\mathrm{W}, \mathrm{V}_{2}\right)=\left(\mathrm{W} / \mathrm{W}_{\mathrm{c}}\right)^{\mathrm{b} \log \left[\mathrm{f}\left(\mathrm{V}_{1}\right) / \mathrm{f}\left(\mathrm{V}_{2}\right)\right]}
$$

and

$$
H\left(W, V_{1}\right) / H\left(W, V_{2}\right)=\left[f\left(V_{1}\right) / f\left(V_{2}\right)\right]^{\left[1-\left(\log W / \log W_{c}\right)\right]},
$$

in which the constant, or volume independent. terms in Equations 4a and $4 \mathrm{~b}$ cancel.

All that is needed in order to derive an explicit illusion function is the form of the function $f(V)$. A reasonable candidate for $f(V)$, one that is in accord with the data of Stevens and Rubin, as we will show later, is the simple power function

$$
f(V)=d V^{-e} \quad(e>0)
$$

where $d$ and e are empirical constants estimated from the data ( $\mathrm{e}$ is not to be confused with the Naperian constant $\mathrm{e}=2.718 \ldots$... Fries and Holmberg (1967) and Kolehmainen and Mikkonen (1970) both found that for stimuli of constant weight subjective heaviness is a power function of volume. Stevens and Rubin considered this possibility, but a graphical anaiysis of their data revealed a slight curvature in the relation between log heaviness and log volume. Looking at the same data in semilog coordinates, they concluded that, for constant weight, heaviness decreases approximately as a logarithmic function of volume-although they acknowledged that if the heaviness functions were converging power functions, the illusion functions could not be exactly logarithmic. Since the logarithmic function is logically inconsistent with the representation of heaviness by a system of converging power functions of weight and volume and since there is experimental support for the power function, it will be used here.

Substituting Equation 6 in Equation $5 b$ produces the following equation for the size-weight illusion:

$$
\mathrm{H}\left(\mathrm{W}, \mathrm{V}_{1}\right) / \mathrm{H}\left(\mathrm{W}, \mathrm{V}_{2}\right)=\left(\mathrm{V}_{1} / \mathrm{V}_{2}\right)^{-\mathrm{e}\left[1-\left(\log \mathrm{W} / \log \mathrm{W}_{\mathrm{c}}\right)\right]} \text {. }
$$

This is a quantitative illusion function which tells how much heavier one weight seems than another which has the same mass but a different volume; the heaviness ratio is a power function of the volume ratio. Only two free parameters are involved in this function: the exponent $e$ of the power function given in Equation 6 and the weight $\mathbf{W}_{c}$ which Stevens and Rubin interpret as the heaviest weight that can be confortably lifted in the experiment. This is a natural interpretation of $W_{c}$. which suggests that it should vary with the mechanics of the task; smaller values of $W_{c}$ are to be expected if, for example, the subject must lift the object by gripping an attached knob with the fingers of one hand (as in the Stevens-Rubin experiment), and a higher value of $W_{c}$ should obtain when the object can be seized firmly with both hands and hefted.

Both the direction and the magnitude of the illusion can be derived from Equation 7, with the following consequences and predictions: (a) $\mathrm{H}\left(\mathrm{W}, \mathrm{V}_{1}\right)>$ $\mathrm{H}\left(\mathrm{W}, \mathrm{V}_{2}\right)$ if $\mathrm{V}_{1}>\mathrm{V}_{2}$ and $\mathrm{W}<\mathrm{W}_{\mathrm{c}}$; (b) $\mathrm{H}\left(\mathrm{W}, \mathrm{V}_{1}=\right.$ $\mathrm{H}\left(\mathrm{W}, \mathrm{V}_{2}\right)$ if either $\mathrm{V}_{1}=\mathrm{V}_{2}$ or $\mathrm{W}=\mathrm{W}_{\mathrm{c}}$; (c) The magnitude of the illusion increases with an increase in relative volume of the compared objects (i.e., with an increase in the size of the ratio $V_{1} / V_{2}$ ); (d) the magnitude of the illusion decreases as the equal weight of the compared objects increases; and (e) the magnitude of the illusion increases with $W_{c}$. All of these consequences, with the exception of (e), were observed by Stevens and Rubin.

The present study was designed to further test the validity of the assumptions leading to these predictions, to partially replicate the original study of Stevens and Rubins which provided the fundamental insights enabling the derivation of Equation 7, and, most importantly, to see if $\mathrm{W}_{\mathrm{c}}$ can be increased by modifcation of the lifting task with the consequence of enhancing the size of the illusion.

\section{METHOD}

\section{Stimuli}

The stimuli were 42 cylinders varying in size and weight. They were made from commercial plastic tubing of diameters $4.05,4.6$, $5.5,6.2,7.7,8.6,11.0,12.5$, and $14.5 \mathrm{~cm}$ cut to heights $7.3,8.6$, $10.2,11.5,14.4,17.5,20.3,24.1$, and $28.7 \mathrm{~cm}$ to produce a total of nine sizes: $94,143,239,345,669,1.008,1,943,2,943$, and 
$4.710 \mathrm{cc}$. Polystyrene foam inserts were centrally loaded with varying amounts of lead shot and fitted snugly in to each cylinder in order to produce nine different weights of $51,92,168,308,567$, $1.036,3,480,1.889$, and $6,168 \mathrm{~g}$. Plastic disks were cemented on each end, and the cylinders were then painted a flat black. The combinations of weights and volumes selected for the 42 stimuli are given in Table 1 as the rows and columns that have cell entries. Because of the wide ranges of sizes and weights involved, it was impossible to construct a cylinder with every size-weight combination; e.g.. a 94-cc object weighing $6,168 \mathrm{~g}$ would have a density of 65.6 (lead has a density of 11.3).

\section{Subjects}

Twenty university students. 5 female and 15 male, were selected on the basis of their availability and willingness to serve in this study without pay. None had had any previous laboratory experience requiring psychophysical judgments.

\section{Procedure}

The subjects were run individually in single sessions lasting approximately $35 \mathrm{~min}$. In the beginning of the session, the subject was shown a series of 20 lines varying in five steps from 1.2 to $22.3 \mathrm{~cm}$ in length and was asked to estimate the approximate length of each. The purpose of this exercise was to provide practice making proportional numerical judgments and to insure that the subject understood the instructions for magnitude estimation.

The subject was then told that cylinders of varying size and weight would be presented to him; he was to grasp each one firmly with both hands in the middle of the cylinder, holding it upright, give it a single heft. and judge its "heaviness" by assigning "a number that reflects only the weight of each object and preserves the ratios of weights between objects." No standard stimulus was specified, nor was a modular response required. The subjects were free to use any numbers that seemed appropriate to them.

Stimuli were presented one at a time through a curtained opening of a large white screen that separated the subject from the experimenter. The subject never viewed more than one cylinder at a time.

Most of the 42 stimuli were presented for judgment only once each, but repeated judgments were obtained for two uniform weight series and two uniform volume series. These are the stimuli indicated by asterisked entries in Table 1 . The four stimuli in each of these series were presented four extra times each in a schedule that permitted each separate level in a series to be preceded by each other level exactly once. This was done to facilitate the estimation of order effects in weight judgments. When such effects occur in magnitude estimation experiments, they may contribute to regression biases that affect the exponent of the power function that is fitted to the data (Cross. 1973). The subjects were divided equally into two groups; one group received the extra presentations of stimuli equal in weight but varying in volume (the uniform weight series), and the other group received the extra presentations of stimuli equal in volume but varying in weight (the uniform volume series). Thus, each subject gave a total of 74 weight judgments: one to each of 34 weights and five to each of 8 weights.

\section{RESULTS}

Geometric means of the heaviness estimates were calculated for each cylinger. The results are presented in Table 1. For most entries, the geometric means are based on 20 judgments, 1 from each subject. The asterisked entries are based on 60 judgments, 1 from each of 10 subjects and 5 from each of 10 subjects. The double-asterisked entires are based on 100 judgments, 5 from each of 20 subjects.

The geometric mean heaviness estimates are plotted in $\log -\log$ coordinates as a function of weight in Figure 1. A separate function is depicted for each volume. The horizontal positions of these nine plots have been arbitrarily adjusted for purposes of clarity, but the form and slope of each regression remains unaltered. For each volume series, a separate power function was fitted by the method of least squares, with the results shown by the straight lines. The exponent values and proportionality constants for the power functions represented by these lines in normal (unadjusted) position are given in Table 2. A test for parallelism (Sprent, 1969) indicates that the differences between exponents are significant; $F(8,24)$ $=3.79(\mathrm{p}<.01)$.

If the power functions characterized by the exponents and proportionality constants in Table 2 are convergent (intersect at a common point), then the exponents, $\beta=g(V)$, should be linearly related to the logarithms of the proportionality constants, $\alpha=f(V)$, as expressed by Equation 3. The linear correlation between $\beta$ and $\log \alpha$ for these data is $r=-0.993$, indicating experimental verification of Equation 3 .

Estimation of the point of concurrence $\left(\mathrm{H}_{\mathrm{c}}, \mathrm{W}_{\mathrm{c}}\right)$ for this family of power functions is problematic because of the difficulty of getting unbiased estimates of the regression parameters in Equation 3. Both $\beta$ and $\alpha$ are estimated with error, hence the regression of $\beta$ on $\log$ $\alpha$ and the regression of $\log \alpha$ on $\beta$ are both biased estimates of the true regression parameter (Isaac, 1970).

Table 1

Magnitude Estimations of the Heaviness of Plastic Cylinders Varying in Weight and in Volume

\begin{tabular}{|c|c|c|c|c|c|c|c|c|c|}
\hline \multirow{2}{*}{$\begin{array}{c}\text { Weight } \\
\text { (g) }\end{array}$} & \multicolumn{9}{|c|}{ Volume (cc) } \\
\hline & 94 & 143 & 239 & 345 & 669 & 1008 & 1943 & 2943 & 4710 \\
\hline 6168 & & & & & & & 49.3 & $48.7^{*}$ & 44.7 \\
\hline 3480 & & & & & & $34.5^{*}$ & $30.6^{*}$ & $27.6^{* *}$ & $25.2 *$ \\
\hline 1889 & & & & & 17.2 & 14.6 & 13.2 & $12.7^{*}$ & 12.8 \\
\hline 1036 & & & 14.5 & 12.6 & 10.5 & 8.6 & 8.4 & $6.1^{*}$ & 4.4 \\
\hline 567 & & & 7.7 & 7.0 & 5.0 & 4.8 & 2.9 & & \\
\hline 308 & 5.1 & $4.9^{*}$ & 4.0 & 4.1 & 2.7 & 2.0 & & & \\
\hline 168 & 3.1 & $3.0^{*}$ & 2.6 & 2.2 & 1.6 & 1.2 & & & \\
\hline 92 & $1.9 *$ & $1.7^{* *}$ & $1.2^{*}$ & $1.0^{*}$ & & & & & \\
\hline 51 & 1.13 & $1.0^{*}$ & & & & & & & \\
\hline
\end{tabular}

Note-Each entry is the geometric mean of 20 estimations (60 for asterisked entries and 100 for doubly asterisked). 


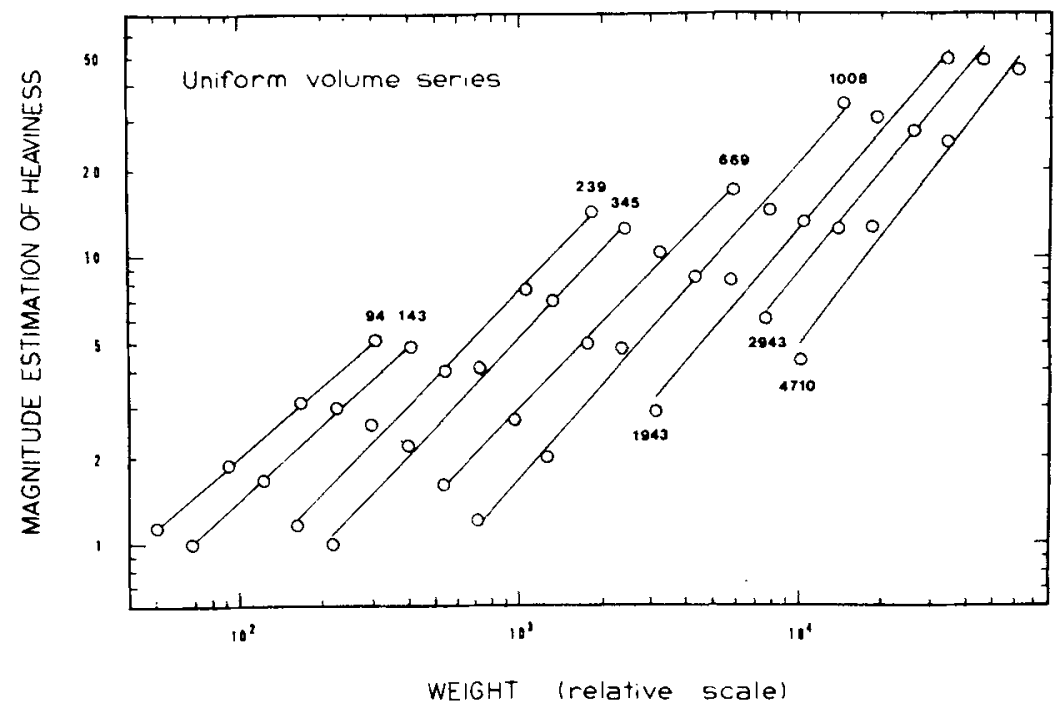

Figure 1. Heaviness as a function of weight. The parameter is volume in cubic centimeters. The horizontal position of each curve has been arbitrarily adjusted for clarity.
Combining Equations 2, 3, and 6 produces the following awkward appearing but explicit relationship between apparent heaviness, $H$, and the stimulus dimensions weight, $W$, and volume, $\mathrm{V}$ :

$$
H(W, V)=d V^{-e} W^{-b e(\log V)+b(\log d)+a} \text {. }
$$

According to this model, the exponents in Table 2 should be linearly related to the logarithm of the corresponding volume, a controlled variable, and the proportionality constants vary as a power function of volume. Unbiased least squares estimates of the parameters $a, b, d$, and e can be obtained from the regression of $\beta$ on $\log V$ and from the regression of $\log \alpha$ on $\log V$ since, according to Equation $8, \beta=$ $-b e(\log V)+b(\log d)+a$ and $\alpha=d^{-e}$. The constant $b$ can be obtained by first solving the second equation for $e$ and dividing this into the proportionality constant of the first equation.

The relation between $\beta$ and $\log V$ for these data is shown in Figure 2. The regression is reasonably linear with $94 \%$ of the variance in the empirical exponent

Table 2

Exponent Values and Proportionality Constants for the Power Functions Found to Relate Heaviness to Weight for Each Volume Series

\begin{tabular}{cccc}
\hline $\begin{array}{c}\text { Volume } \\
\text { (cc) }\end{array}$ & $\begin{array}{c}\text { Propor- } \\
\text { tionality } \\
\text { Constant }\end{array}$ & $\begin{array}{c}\text { Obtained } \\
\text { Exponent } \\
\text { Value }\end{array}$ & $\begin{array}{c}\text { Adjusted } \\
\text { Exponent } \\
\text { Value }\end{array}$ \\
\hline 94 & .0428 & .835 & .945 \\
143 & .0304 & .889 & .999 \\
239 & .0127 & 1.013 & 1.123 \\
345 & .0103 & 1.031 & 1.141 \\
669 & .0087 & 1.010 & 1.120 \\
1008 & .0040 & 1.102 & 1.212 \\
1943 & .0021 & 1.164 & 1.274 \\
2943 & .0018 & 1.176 & 1.286 \\
4710 & .0007 & 1.281 & 1.391 \\
\hline
\end{tabular}

values accounted for by linear regression on $\log \mathrm{V}$. The deviations from linear regression are not significantly different from the weighted average of deviations from linear regression used as an error term in the above test for parallelism: $F(7,24)=2.03$ (n.s.). On the other hand, the linear regression of $\alpha$ on $\log V$ is significant: $F(1,7)=6.89(\mathrm{p}<.05)$.

In the log-log coordinates of Figure 3, the constants of proportionality are plotted as a function of volume. It is clear that the assumption of Equation 6 is reasonably valid for these data: $97.6 \%$ of the variance in $\log \alpha$ is accounted for by linear regression on $\log V$. In this study, the constant of proportionality for each heaviness function turns out to be approximately proportional to the reciprocal of physical volume.

Estimates of the parameters $a, b, d$, and e, derived from these functions, are shown in Table 3 . The point of concurrence for the theoretical family of converging heaviness functions for the experimental conditions of the present study occurs at approximately $18 \mathrm{~kg}$ (about $40 \mathrm{lb}$ ).

When the estimated parameter values are inserted in the illusion function given by Equation 7, the following simplified version results:

$$
\mathrm{H}\left(\mathrm{W}, \mathrm{V}_{1}\right) / \mathrm{H}\left(\mathrm{W}, \mathrm{V}_{2}\right)=\left(\mathrm{V}_{1} / \mathrm{V}_{2}\right)^{0.23(\log \mathrm{W})-0.98} \text {. }
$$

In Figure 4, the geometric mean heaviness estimates are plotted as a function of volume showing the size-weight illusion in log-log coordinates. The parameter is weight in grams. The slopes of the straight lines drawn through each set of points are given by the exponents of Equation 9 for the different weights, $W$.

Figure 4 reveals several features of the size-weight illusion. As predicted by the model, the magnitude of the illusion depends on both size and weight. The effect of size is greater for lighter weights and decreases as the weight of the judged object increases. 


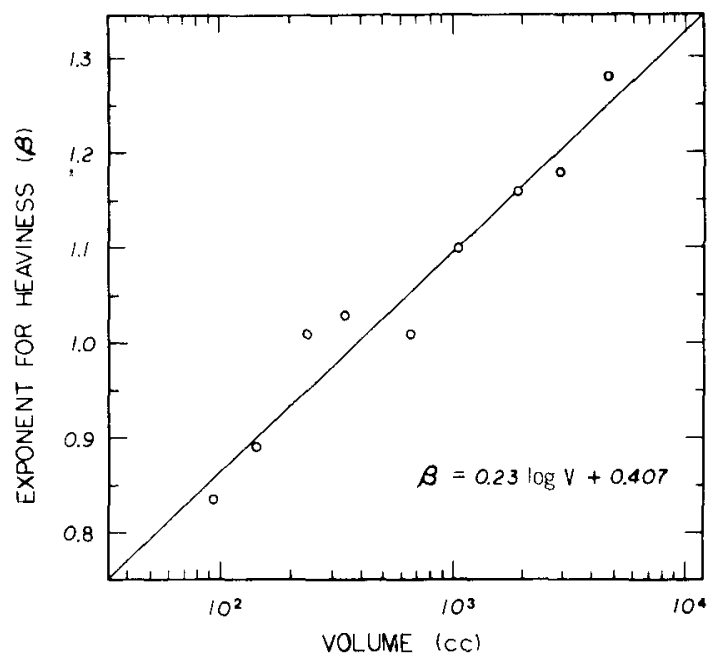

Figure 2. The exponent for heaviness as a function of size.

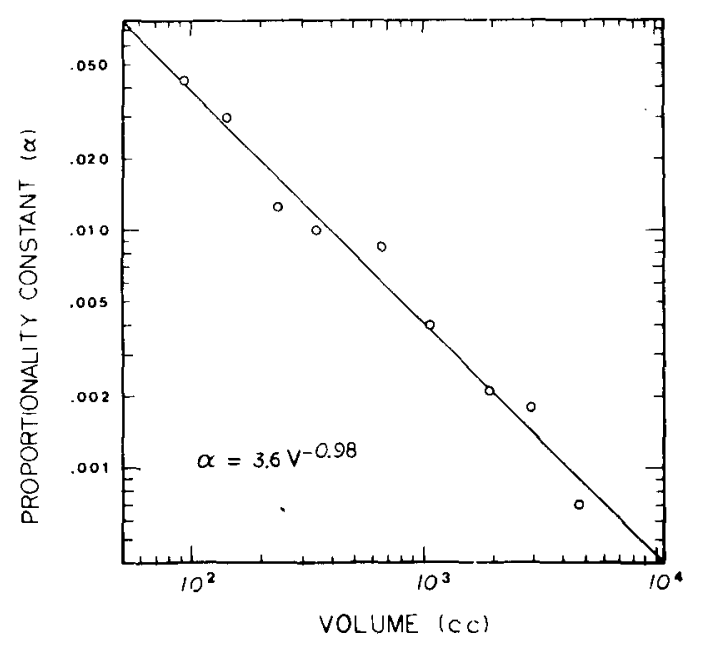

Figure 3. The relation between the proportionality constant in the heaviness function and size.

For a given weight, the greater its volume the lighter it appears to be. The magnitude of the illusion is sufficiently great that one object having twice the physical weight of another may in fact appear lighter if it is four or five times larger in size.

The data of Stevens and Rubin were reanalyzed with the present model in mind, and the resulting estimates for the parameters $a, b, d$, and e are shown in Table 3 for comparison with the results of the present study. The family of power functions describing these data converge at a weight of $8.7 \mathrm{~kg}$. This represents the maximum weight that could be lifted under the conditions of the experiment in which each weight was grasped by an attached knob with the fingers of one hand and lifted. This is less than half the value of $18 \mathrm{~kg}$ obtained in the present study, in which both hands could be used by grasping the cylinders firmly around their middle and hefting them.

The power function of Equation 6 well describes the relation between the proportionality constants of the Stevens-Rubin heaviness functions and volume. For their data, approximately $95.3 \%$ of the variance in $\log \alpha$ is accounted for by linear regression on $\log \mathrm{V}$. However, the exponent of -0.65 is only two-thirds the value obtained in the present study. The difference between these two outcomes is puzzling, and nothing in the differences in experimental procedure suggests itself as a possible explanation for the difference.

The values for the parameters given in Table 3 were inserted in Equation 8, and theoretical heaviness values were computed for the weights used by Stevens and Rubin as well as for those used in the present study. The relation between theoretical (fitted) values and obtained heaviness estimates is shown in Figure 5 for the Stevens-Rubin study and in Figure 6 for the present study. The agreement between theoretical and obtained values is good in both cases. Only $0.3 \%$ of the variance of obtained estimates remains unaccounted for by the model in the first study and $0.7 \%$ is unaccounted for in the present study.

The exponents of the heaviness functions shown in Table 2 are systematically lower than those obtained by Stevens and Rubin for comparable volumes. These differences can be attributed, in part, to differences in the magnitude of the size-weight illusion in the two studies (different values of $W_{c}$ and $e$ ). On the other hand, cross-modality matching procedures in general and magnitude estimation procedures in particular are known to be subject to regression biases that cause the theoretically correct exponents to be underestimated (S. S. Stevens \& Greenbaum, 1966). The possibility of regression bias in the present data cannot be ignored.

Two types of regression bias have been identified. One results in a multiplicative transformation of the correct exponent (Cross, 1974). This is interpreted as a response bias that arises from tendencies to avoid extreme judgments or to be limited in the range of responses available (as in category scaling). If this source of bias is operating in the present experiment, it is as likely to influence the estimation of line length as it is to bias the heaviness judgments. The line

Table 3

Evaluation of Parameters: Results of Two Studies

\begin{tabular}{ccc}
\hline $\begin{array}{c}\text { Para- } \\
\text { meter }\end{array}$ & $\begin{array}{c}\text { Present } \\
\text { Study }\end{array}$ & $\begin{array}{c}\text { Stevens and } \\
\text { Rubin (1970) }\end{array}$ \\
\hline a & .5407 & .721 \\
b & -.235 & -.254 \\
e & .98 & .65 \\
d & 3.66 & .97 \\
W $_{\mathrm{c}}$ & $18 \mathrm{~kg}$ & $8.7 \mathrm{~kg}$ \\
$\mathrm{H}_{\mathrm{c}}$ & 200 & 690 \\
\hline
\end{tabular}




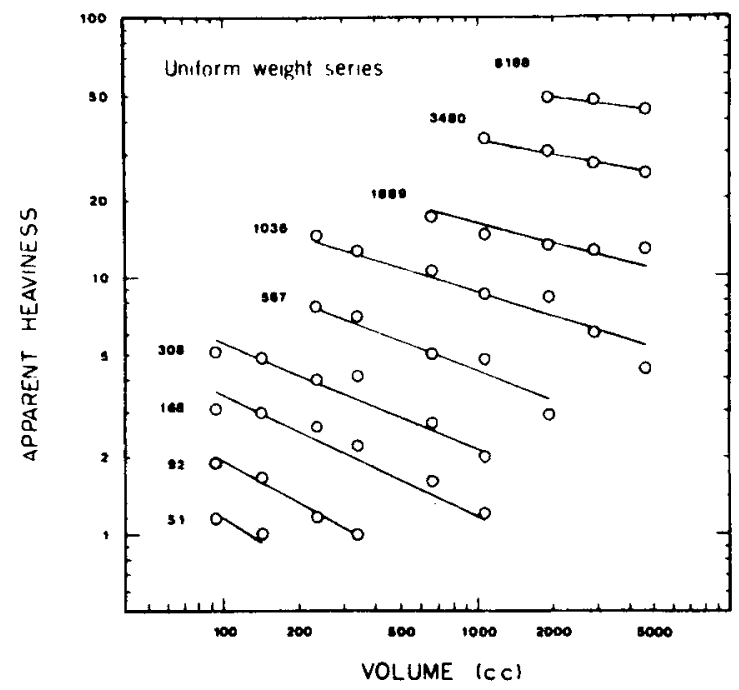

Figure 4. Heaviness as a function of volume showing the size-weight illusion in log-log coordinates. The parameter is weight in grams. The lines have the slopes given by the illusion function of Equation 7.

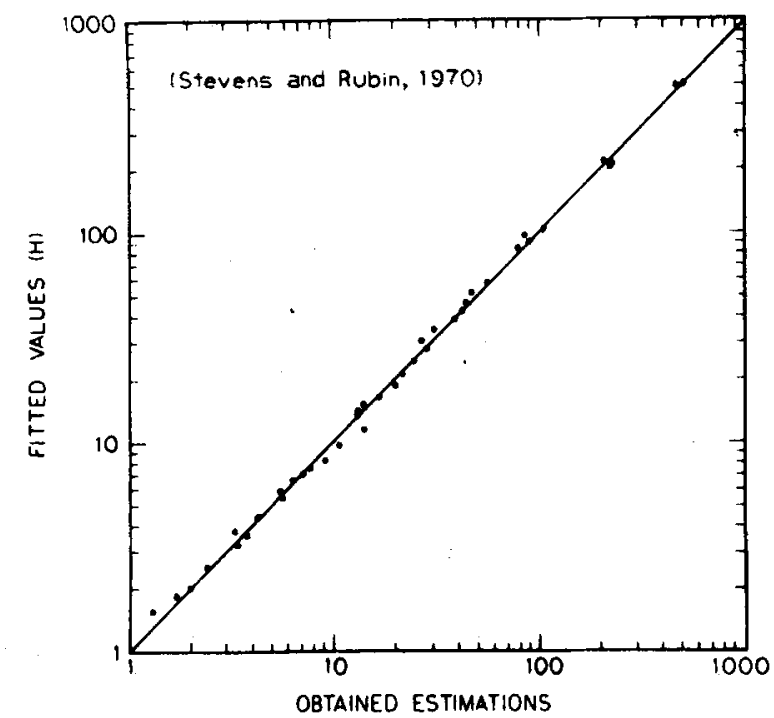

Figure 5. Fitted values of heaviness given by Equation 8 with the parameter values shown in Table 3 vs. obtained heaviness estimations (data from Stevens \& Rubin, 1970).

length judgments obtained in the beginning of the experimental sessions of the present study were averaged over subjects and regressed against objective length. A power function with an exponent equal to 0.884 was found to describe the relation between geometric mean length estimates and physical length. Although $99.5 \%$ of the variance in log line judgments were accounted for by linear regression on the logarithm of actual length, the regression coefficient 0.884 is not significantly different from the expected value of unity. Therefore, it is probably not appropriate to conclude that response biases are responsible for the lower exponents in the heaviness functions.

The other contribution to regression arises from order effect in the perception of temporally ordered stimuli. This contribution to regression adds or subtracts a constant to the correct exponent, depending on whether the order effects are contrastive or assimilative. It appears that in magnitude estimation tasks, order effects are usually assimilative and contribute to an underestimation of the true exponent (Cross, 1973; Luce \& Green, 1974). In this case, it may be possible to represent perceived magnitude as a power product of the focal stimulus and the immediately preceding stimulus as follows:

$$
\Psi=\mathrm{kS}_{\mathbf{i}}^{\beta-\mathrm{o}} \mathrm{S}_{\mathbf{i}-\mathbf{1}}^{\mathrm{o}},
$$

where $S_{i}$ and $S_{i-1}$ are the stimuli presented on Trials $i$ and $i-1, \beta$ is the true exponent, and $o$ is a bias parameter reflecting the size of the order effect. When each stimulus level is preceded by every other one, the geometric mean of the biased estimates of stimulus magnitude will be proportional to the focal stimulus intensity raised to the power $\beta-o$. Hence, the exponent estimated by conventional methods underestimates the true exponent by an amount equal to $o$. In order to remove this source of bias from estimates of exponent values, the order effect must be measured.

Figure 7 shows that assimilative order effects were indeed present in this study. For the two uniform volume series of weights in which a counterbalanced presentation schedule allowed each weight in the series to be preceded by every other weight, including

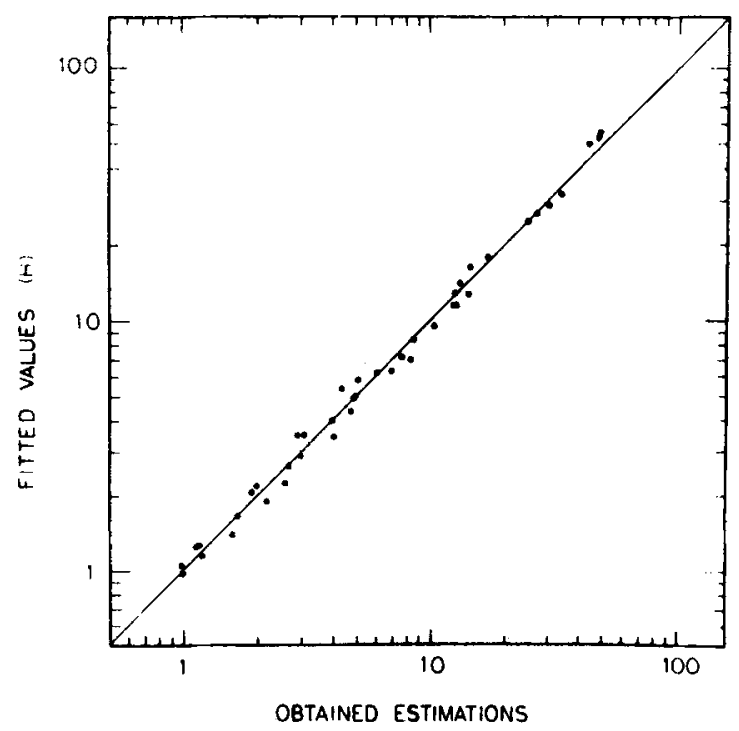

Figure 6. Fitted values of heaviness given by Equation 8 using the parameter values shown in Table 3 for the data of the present study. 
itself, the apparent heaviness of each object is shown as a function of the weight of the immediately preceding object (filled circles) and, separately, as a function of the weight in grams of the focal object (open circles). The open circles represent the geometric means of the four filled circles for each weight of the focal stimulus which is also shown as a parameter for the family of functions depicted in Figure 7. These data are based on the judgments of 10 subjects. Each filled circle in Figure 7 represents the geometric mean of 10 independent judgments. The dashed lines drawn through each set of four points represents the average effect of the preceding stimulus level on estimates of the heaviness of the focal stimulus where the average is taken over all levels of the focal stimulus for both uniform volume series. The slope of these dashed lines corresponds to the bias exponent. $o$, in Equation 10. For these data, o has an estimated value of 0.11 .

A second group of 10 subjects gave repeated heaviness judgments to two uniform weight series in which the stimuli were equal in weight and they varied in volume. Although apparent heaviness varies with volume (see Figure 4), successive jud gments to stimuli varying only in volume showed no order effect. The average value of o for these data turned out to be practically zero $(0=0.008)$.

Since order effects are presumably present only with sequential variations in weight and not in volume, the effects of this bias can be eliminated by adding the constant amount 0.11 to the exponent for weight, $\mathrm{W}$, in Equation 8. This increases each of the obtained exponents listed in Table 2 by the amount 0.11 hence the true exponents are estimated to vary between 0.94 for the 94 -ce cylinders to 1.39 for the $4,710-\mathrm{cc}$ cylinders), but only the experimental estimate for the parameter $a$ is affected by the adjustment. Parameters $\mathrm{b}, \mathrm{d}$, and $\mathrm{e}$ remain unchanged. This means that the point of convergence of the heaviness functions remains at $18 \mathrm{~kg}$, but the estimate for $\mathrm{H}_{\mathrm{c}}$ increases from 200 to 590 corresponding to the increase in a from 0.5407 to 0.6507 .

\section{DISCUSSION}

Over the years, many explanations have been offered for the size-weight illusion. Some accounts have been based on set or expectation and consequent motor adjustment to that expectation (Loomis, 1907; Woodworth, 1921). Other theories have identified density as either the effective stimulus for judgments of heaviness or as having a biasing influence on such judgments (Huang, 1945; Ross \& DiLollo, 1970; Seashore, 1913).

A third type of explanation is based on information integration (Anderson, 1970, 1972; Birnbaum \& Veit,

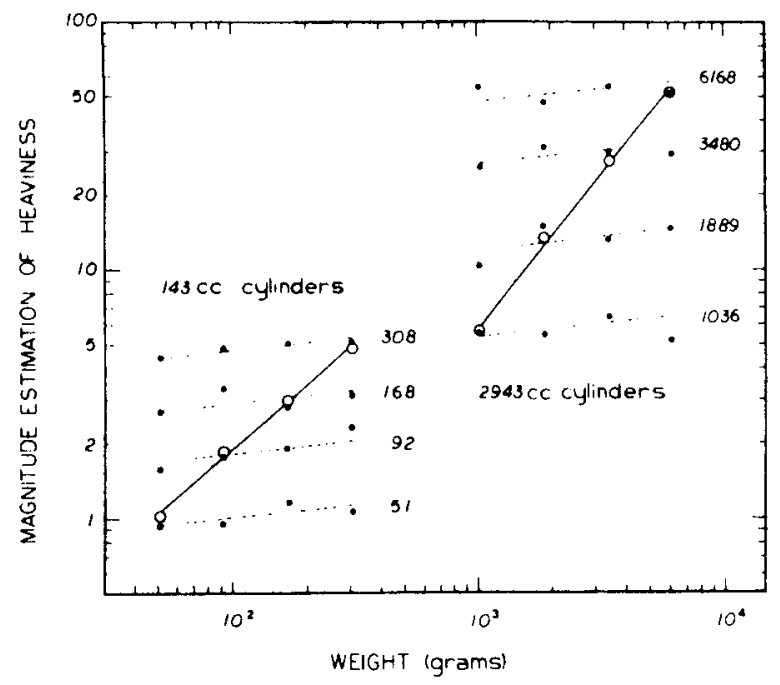

Figure 7. Apparent heaviness for two sizes of weights as a function of weight in grams of the focal object (open circles) and as a function of the weight of the immediately preceding object (filled circles). The parameter is the weight of the focal object. The dashed lines have a common slope equal to 0.11 .

1974); Sjöberg, 1969). According to this theory, the perception of a stimulus depends on the integration of separate bits of information about it. In the case of weight, "we have an interaction of visual and kinesthetic inputs which produce a final percept of heaviness which depends on both" (Sjöberg, 1966, p. 132). The difference between the theories of this kind resides chiefly in whether the effects of size and weight are assumed to combine additively (Anderson, 1970) or multiplicatively (Sjöberg, 1969).

These explanations are either substantially incorrect or they are incomplete in that they leave various features of the size-weight illusion unexplained. Traditional expectation theoriès do not explain the consistent decrease in the magnitude of the illusion with increasing weight found by J. C. Stevens and Rubin (1970) and in the present study. Density interpretations do not explain differences in muscle effort (Loomis, 1907) or muscle potentials prior to the lift (Jarrard, 1960; Payne \& Davis, 1940). Nor can density effects explain the differences in judged heaviness found by Harshfield and De Hardt (1970), whose stimuli were of identical size and weight, and differed only in apparent composition, e.g., steel vs. balsa wood.

The information integration models are wrong because, in their simple versions, they assume that the magnitude of the illusion for any two weights is independent of size, and for any two sizes is independent of weight (Birnbaum \&Veit, 1974).

The model proposed here handles all the established facts concerning the size-weight illusion. In the one possibly discrepant study, Ross and DiLollo (1970) propose a variable-basis judgment 
model in which heaviness judgments are increasingly based on density as stimulus density increases. They report the finding that for light weights differences in weight produce large judged heaviness differences, while differences in density produce lesser differences in heaviness. For heavy weights, changes in weight and density produce identical differences in judged heaviness. However, it is possible that the subjects were not discriminating weight differences in the heavy weights because of the small range of differences employed (less than $25 \%$ of the maximum weight). The most outstanding of their results is that while there was a substantial illusion for light weights, for heavy weights the illusion was virtually nonexistent, a result that is predicted by the present model.

None of the previous theories take into account the finding that the size of the illusion may be affected by the way the weights are lifted. We predicted that the change in the manner of lifting the weights would lead to an increase in the magnitude of the illusion by increasing the size of the maximum effective weight that can be comfortably lifted. That the slopes of the illusion functions shown in Figure 4 are steeper than the functions for comparable weights found by Stevens and Rubin confirms this prediction.

Other factors may be expected to influence the size of this illusion by alteration of the point of convergence of the heaviness functions. Men, being stronger in general than women and thus able to lift heavier weights, should exhibit a greater illusion because of an increase in $W_{c}$. By the same reasoning, young children should show less of the size-weight effect than older children. The magnitude of the illusion should increase with age. The experimental facts indicate just the opposite. Numerous studies involving children as experimental subjects show a decrease in the magnitude of the illusion with age (Oöstlander, 1967; Pick \& Pick, 1967; Robinson, 1964). This decrease seems to be rapid until about age 11 and then levels off. The illusion is shown by children as young as $1 \frac{1}{2}$ years of age, decreases with age and, presumably, experience. Of course, other things are not equal in these comparisons. The discrepancy may be explained by poor weight discrimination. Robinson (1964) showed that with weight discrimination training, the magnitude of the illusion in children decreased. Adults may simply discern weights better than children.

J. C. Stevens (1974) has shown that there are many sensory modalities, in addition to the proprioceptive senses, in which families of converging power functions describe the interaction between component properties of a stimulus. The relation between brightness and luminance varies as a function of level of light adaptation, of glare angle, and of the size of an inhibitory surround. The perception of warmth as a function of irradiation varies with areal extent; the perception of cold varies with duration. Auditory volume and density, as functions of sound pressure, both also vary with frequency of the tone. In each of these cases, and others, the relations observed can be described by power functions that converge at a common point. In some cases, this common point corresponds to a level of stimulation that coincides with the threshold of pain or the limits of sensory functioning for the modality.

The similarity between the operating characteristics of these sensory systems and that of the size-weight interaction in the perception of heaviness causes us to question the appropriateness of classifying this effect as an illusion. Consider, for example, the well-known dependence of loudness on tonal frequency as well as intensity. Equal-loudness contours drawn in sound-pressure/frequency coordinates tend to converge for frequencies below $400 \mathrm{~Hz}$ because the exponent of the power function that governs loudness increases very rapidly as frequency decreases (S. S. Stevens, 1972). We could construct a set of equal-heaviness contours in the coordinate space of size and weight. These contours would be similar in appearance (although converging to the right instead of to the left) to the equal-loudness contours for low-frequency tones. The slope of the equal-heaviness contours increases as level decreases. The contours reveal that the heaviness of a weight depends on its size. Are these statements about heaviness really any different from the analogous statements about loudness; namely, that the equal-loudness contours are steeper for softer tones, and the loudness of a tone depends on its frequency? We think of the size-weight effects as illusory, but the interaction between sound pressure and frequency in loudness determination is said to be only an operating characteristic of the auditory system. When the same relations observed for weights are observed in other sensory systems, we do not typically think of the perceived effects as illusory, nor do we seek psychological explanations for the effects. Perhaps the same attitude should be taken toward the interacting roles played by size and weight in the determination of perceived heaviness. Just as the loudness of a tone varies with frequency, the heaviness of a weight depends on its size. Heaviness can be characterized by the system of converging power functions for the variables of size and weight just as loudness is characterized by a similar set of relations defined for frequency and sound pressure.

\section{REFERENCES}

Anderson, N. H. Averaging model applied to the size-weight illusion. Perception \& Psychophysics, 1970, 8, 1-4.

ANDERson, N. H. Cross-task validation of functional measurement. Perception \& Psychophysics, 1972, 12, 389-395.

Birnbaum, M. H., \& Veit, C. T. Scale free tests of an additive model for the size weight illusion. Perception \& Psychophysics, 1974, 16, 276-282. 
Cross, D. V. Sequential dependencies and regression in psychophysical judgments. Perception \& Psychophysics, 1973 , 14. 547-552.

Cross, D. V. Some technical notes on psychophysical scaling. In H. R. Moskowitz, B. Scharf, \& J.C. Stevens (Eds.), Sensation and measurement: Papers in honor of S. S. Stevens. Dordrecht-Holland: Reidel, 1974. Pp. 23-36.

FrIEs, 1., \& Holmberg, L. The psychophysics of the size-weight illusion. I. Functional relation between heaviness and volume estimations. Psychological Research Bulletin (Lund University, Sweden), 1967, 7, 1-20.

Harshfield, S. P., \& DeHardt, D. C. Weight judgment as a function of apparent density of objects. Psychonomic Science, 1970. 20. 365-366.

Huang, I. The size-weight illusion and the "weight-density illusion." Journal of General Psychology, 1945, 33, 65-84.

ISAAC, $P$. D. Linear regression, structural relations, and measurement error. Psychological Bulletin. 1970, 74, 213-218.

JARRARD, L. E. The role of visual cues in the performance of ergographic work. Journal of Experimental Psychology, 1960. 60. 57-63.

Kolehmainen, K., \& Mikkonen, V. Apparent weight differences as a source of after-effects. Scandinavian Journal of Psychology, 1970. 11, 161-166.

Loomis. H. N. Reactions to equal weights of unequal size. Psychological Monographs, 1907, 8, 334-348.

LUCE, R. D., \& GREEN, D. M. The response ratio hypothesis for magnitude estimation. Journal of Mathematical Psychology. $1974,11,1-14$.

Oöstlander, A. M. The development of the weight-volume illusion. Journal of Experimental Child Psychology, 1967, 5, 237-248.

PAYNE, B., \& Davis, R. C. The role of muscular tension in the comparison of lifted weights. Journal of Experimental Psychology, 1940, 27, 227-242.

Pick. H. L., \& Pick, A. D. A developmental and analytic study of the size-weight illusion. Journal of Experimental child Psychology, 1967, 5, 362-371.
Robinson, H. B. An experimental examination of the size-weight illusion in young children. Child Development, 1964, 35. 91-107.

Ross, J., \& Dilollo, V. Differences in heaviness in relation to density and weight. Perception \& Psychophysics, 1970, 7, 161-162.

Seashore, C. E. Psychology in daily life. New York: Appleton, 1913.

SuöвERG, L. A method for sensation scaling based on an analogy between perception and judgment. Perception \& Psychophysics, 1966, 1, 131-136.

SJöberg. L. Sensation scales in the size-weight illusion. Scandinavian Journal of Psychology, 1969, 10. 109-112.

SPRENT, P. Models in regression and related topics. London: Methuen, 1969.

Stevens, J. C. Families of converging power functions in psychophysics. In H. R. Moskowitz, B. Scharf, \& J. C. Stevens (Eds.), Sensation and measurement: Papers in honor of $S$. $S$. Stevens. Dordrecht-Holland: Reidel, 1974. Pp. 37-47.

Stevens, J. C., \& Rubin, L. L. Psychophysical scales of apparent heaviness and the size-weight illusion. Perception \& Psychophysics, 1970, 8, 225-230.

Stevens. S. S. Perceived level of noise by Mark VII and decibels (E). Journal of the Acoustical Society of America, 1972. 51. 575-601.

Stevens, S. S., \& Galanter, E. Ratio scales and category scales for a dozen perceptual continua. Joumal of Experimental Psychology, 1957, 54, 377-411.

Stevens, S. S., \& Greenbaum, H. B. Regression effects in psychophysical judgment. Perception \& Psychophysics, $1966,1,439-446$.

Woodworth, R. S. Psychology: A study of mental life. New York: Holt, 1921.

(Received for publication January 6, 1975; revision received May 21, 1975.) 